Research Article

\title{
Neuropeptides as the Shared Genetic Crosstalks Linking Periodontitis and Major Depression Disorder
}

\author{
Changsheng Sun $\mathbb{D},{ }^{1}$ Jiatong Han $\mathbb{D},{ }^{1}$ Yixin Bai $\mathbb{D},{ }^{2}$ Zhaowei Zhong $\mathbb{D},{ }^{2}$ Yingtao Song $\mathbb{D},{ }^{2}$ \\ and Yu Sun iD ${ }^{2}$ \\ ${ }^{1}$ Department of Oral and Maxillofacial Surgery, Dental Hospital, The First Affiliated Hospital of Harbin Medical University, \\ 23 Youzheng Road, Nangang District, Harbin, 150001 Heilongjiang, China \\ ${ }^{2}$ Department of Prosthodontics, Dental Hospital, The First Affiliated Hospital of Harbin Medical University, 23 Youzheng Road, \\ Nangang District, Harbin, 150001 Heilongjiang, China
}

Correspondence should be addressed to Yu Sun; sunyu20060212@126.com

Received 4 September 2021; Accepted 1 October 2021; Published 21 October 2021

Academic Editor: Yuzhen Xu

Copyright (c) 2021 Changsheng Sun et al. This is an open access article distributed under the Creative Commons Attribution License, which permits unrestricted use, distribution, and reproduction in any medium, provided the original work is properly cited.

\begin{abstract}
Background. The aim of this study was at investigating the association between major depressive disorder (MDD) and periodontitis based on crosstalk genes and neuropeptides. Methods. Datasets for periodontitis (GSE10334, GSE16134, and GSE23586) and MDD (GSE38206 and GSE39653) were downloaded from GEO. Following batch correction, a differential expression analysis was applied (MDD: $|\log 2 \mathrm{FC}|>0$ and periodontitis $|\log 2 \mathrm{FC}| \geq 0.5, p<0.05$ ). The neuropeptide data were downloaded from NeuroPep and NeuroPedia. Intersected genes were potential crosstalk genes. The correlation between neuropeptides and crosstalk genes in MDD and periodontitis was analyzed with Pearson correlation coefficient. Subsequently, regression analysis was performed to calculate the differentially regulated link. Cytoscape was used to map the pathways of crosstalk genes and neuropeptides and to construct the protein-protein interaction network. Lasso regression was applied to screen neuropeptides, whereby boxplots were created, and receiver operating curve (ROC) analysis was conducted. Results. The MDD dataset contained 30 case and 33 control samples, and the periodontitis dataset contained 430 case and 139 control samples. 35 crosstalk genes were obtained. A total of 102 neuropeptides were extracted from the database, which were not differentially expressed in MDD and periodontitis and had no intersection with crosstalk genes. Through lasso regression, 9 neuropeptides in MDD and 43 neuropeptides in periodontitis were obtained. Four intersected neuropeptide genes were obtained, i.e., ADM, IGF2, PDYN, and RETN. The results of ROC analysis showed that IGF2 was highly predictive in MDD and periodontitis. ADM was better than the other three genes in predicting MDD disease. A total of 13 crosstalk genes were differentially coexpressed with four neuropeptides, whereby FOSB was highly expressed in MDD and periodontitis. Conclusion. The neuropeptide genes ADM, IGF2, PDYN, and RETN were intersected between periodontitis and MDD, and FOSB was a crosstalk gene related to these neuropeptides on the transcriptomic level. These results are a basis for future research in the field, needing further validation.
\end{abstract}

\section{Background}

Major depressive disorder (MDD) is a prevalent disease worldwide, showing a prevalence around 5\% and an incidence of approximately $3 \%$, with differences between countries [1]. Thereby, MDD is the most prevalent mental disorder in the world [2]. MDD is a disabling condition, worsening quality of life, limiting patients in their everyday activities, and resulting in a remarkable morbidity of patients [2]. Additionally, MDD is often related with comorbidities, worsening the general outcome of respective patients [2]. It has been indicated that MDD is potentially related to changes in numerous biological pathways and systems, including the gastrointestinal tract, immune system, 
hormones, and oxidative stress [3,4]. In this context, an increased understanding of the etiopathogenesis of MDD and related cofactors is of high clinical interest to reveal potential therapeutic strategies in the future [3]; however, it is difficult to reveal any causalities between other diseases, biomarkers, and MDD in the clinical context [5].

One potentially associated disease is periodontitis, which is an inflammatory, multifactorial infectious disease of the tooth-surrounding tissues $[6,7]$. Generally, severe periodontitis affects about $11 \%$ of the world population, making it a highly prevalent chronic disease [8]. Different systematic reviews and meta-analyses are available, which indicated an association between periodontitis and MDD, although results are quite heterogeneous regarding the magnitude of correlation $[6,9,10]$. A Brazilian birth cohort study revealed a risk ratio of patients with MDD to suffer from periodontitis of 1.19 [11]. Another observational study including 60000 individuals over a 10-year follow-up showed a higher incidence of MDD in periodontally diseased individuals, supported by a hazard ratio of 1.73 [12]. Thereby, it is of interest, whether there is a primary association between periodontitis and MDD based on psychosocial mechanisms or even a causal link [13]. The potential role of neuroinflammation induced by chronic inflammatory periodontal diseases, especially due to the expression of proinflammatory cytokines, has been discussed as potential causal link [13, 14]. Thereby, a role of periodontal pathogenic bacteria has been presumed, which is supported by the induction of neuroinflammation by Fusobacterium nucleatum [15].

Although these approaches are interesting and appear plausible, there is more research needed to support the causal interlink between periodontitis and MDD [13]. One potential approach could be the application of bioinformatics to reveal a potential interlink on the transcriptomic level; this has been established for other potential interlinks, e.g., between periodontitis and Alzheimer's disease [16]. Thereby, the potential integration of crosstalk genes, alongside with neuropeptides could be a promising approach to gain insight into the interrelationship between MDD and periodontitis. Thereby, neuropeptides could be of particular interest, because they are involved in a magnitude of processes and were not examined in the context of MDD and periodontitis, yet. Accordingly, this current study is aimed at investigating the association between MDD and periodontitis based on crosstalk genes and their potential link to neuropeptides in these two diseases.

\section{Materials and Methods}

2.1. Data Download. The expression data of periodontitis and MDD was downloaded from GEO (https://www.ncbi .nlm.nih.gov/geo/). For periodontal disease (PD), gingival tissue samples were used, whereby datasets GSE10334, GSE16134, and GSE23586 were obtained. Whole-blood peripheral blood mononuclear cell (PBMC) samples were used for MDD, where two datasets, i.e., GSE38206 and GSE39653, are obtained. For the dataset GSE38206, the experimental group id MDE-P-0W and the control group id C-OW were included. Data from the experimental group
TABLE 1: Datasets for analysis.

\begin{tabular}{lccccc}
\hline Disease & Series & Platforms & Case & Control & Total \\
\hline \multirow{2}{*}{ MDD } & GSE38206 & GPL13607 & 9 & 9 & 18 \\
& GSE39653 & GPL10558 & 21 & 24 & 45 \\
\hline \multirow{2}{*}{ PD } & GSE10334 & GPL570 & 183 & 64 & 247 \\
& GSE16134 & GPL570 & 241 & 69 & 310 \\
& GSE23586 & GPL570 & 3 & 3 & 6 \\
\hline
\end{tabular}

and control group at 8 weeks of follow-up were not used. For GSE39653, MDD and healthy control (HC) samples were included. The datasets of MDD and PD are shown in Table 1.

2.2. Data Preprocessing and Differential Expression Analysis. Firstly, a probe ID was converted into Gene Symbol according to their platform information. For the data of multiple probes corresponding to the same gene, the mean value of the sample was used as its expression value.

Then, all samples from each disease were combined for $\mathrm{PD}$ and MDD expression profiles. To reduce the differences of included samples, a batch correction using the ComBat method in the "sva" package of R project was applied.

The "limma" of $\mathrm{R}$ language was used for differential expression analysis of the corrected MDD and PD datasets. For MDD, the genes with a $p$ value $<0.05$ and $|\log 2 \mathrm{FC}|>$ 0 were differentially expressed genes (DEGs). Thereby, upregulation was evaluated if $\log 2 \mathrm{FC}>0$, while $\log 2 \mathrm{FC}<$ 0 was the cutoff for downregulated genes. The genes with a $p$ value $<0.05$ and $|\log 2 \mathrm{FC}| \geq 0.5$ in PD were considered as differentially expressed genes. The $\log 2 \mathrm{FC} \geq 0.5$ were the upregulated genes and $\log 2 \mathrm{FC} \leq-0.5$ were the downregulated genes.

2.3. Neuropeptide Download. The neuropeptide data were downloaded from the database NeuroPep (http://www .neuropeptides.nl/) and NeuroPedia (http://proteomics.ucsd .edu/Software/NeuroPedia.html). After merging the two databases, a total of 102 neuropeptide genes were obtained.

2.4. Crosstalk Gene Screening. The intersection of differentially expressed genes obtained from MDD and PD was taken, while the intersection genes were the potential crosstalk genes. To analyze the function of these crosstalk genes, clusterProfiler in R language was used for GO Biological Process and KEGG Pathway enrichment analysis and functions with $p$ value $<0.05$ were significant.

2.5. Differential Coexpression Regulation Links of Crosstalk Genes and Neuropeptides. First, it was analyzed whether there were crosstalk genes within the neuropeptides. This step revealed that none of the genes was both crosstalk gene and neuropeptide. Then, the correlation between neuropeptides and crosstalk genes in MDD and PD was analyzed. Therefore, Pearson correlation coefficient was calculated to assess the direct correlation between neuropeptides and crosstalk genes. The crosstalk gene-neuropeptide pair with the correlation coefficient (CC) absolute value greater than $0.5(|\mathrm{CC}|>0.5)$ was considered as significant relationship 


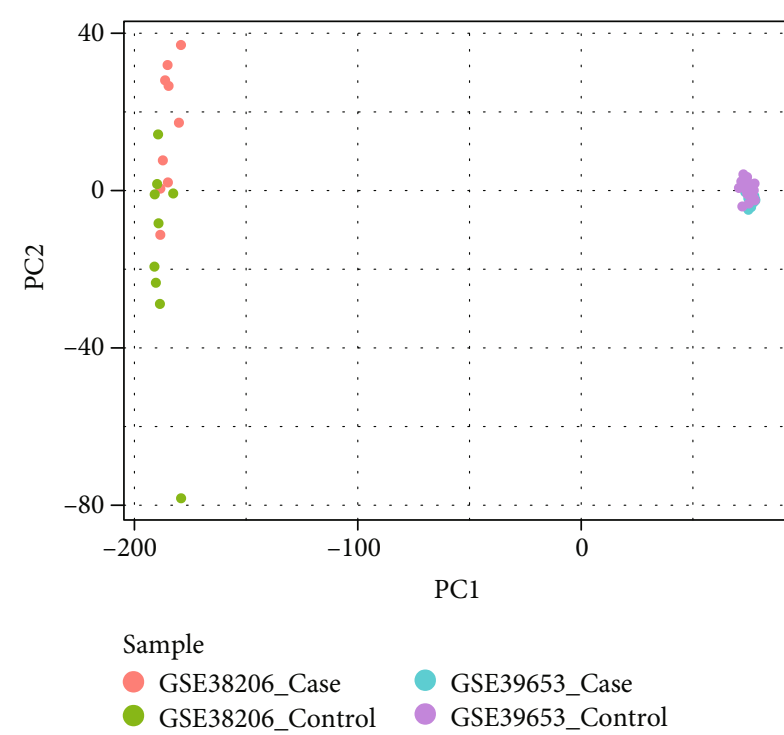

(a)

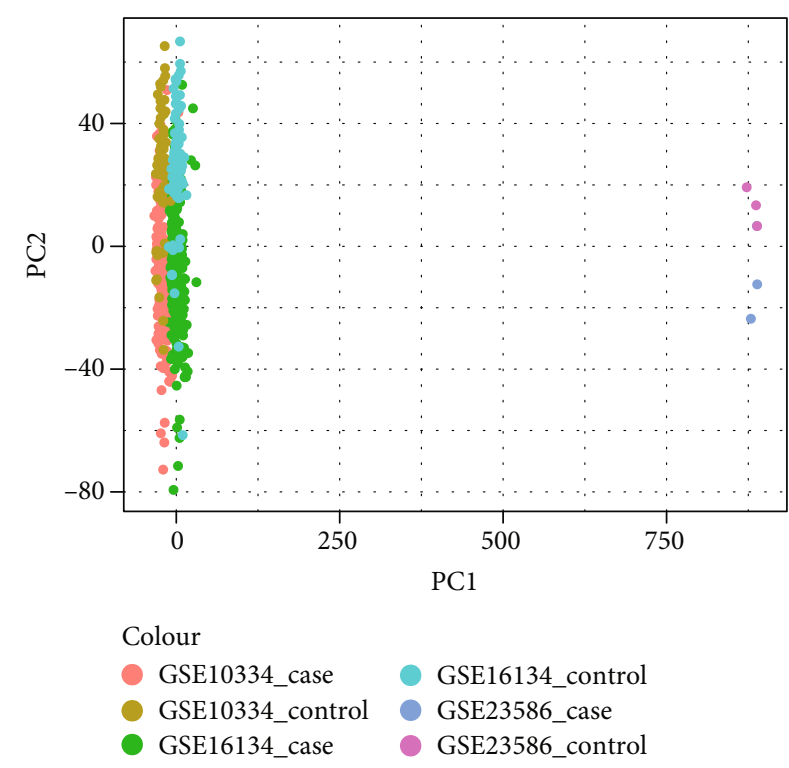

(c)
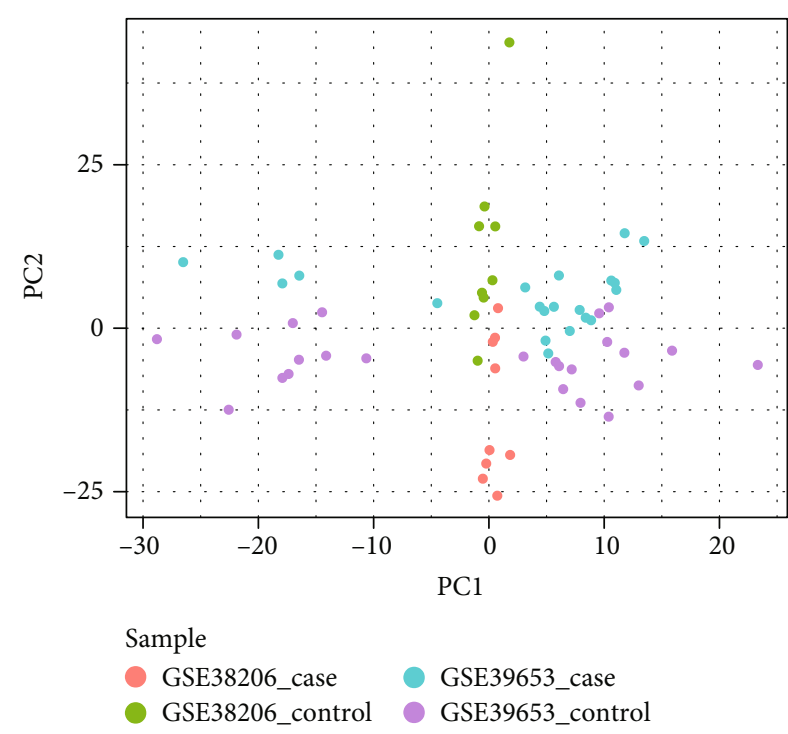

(b)
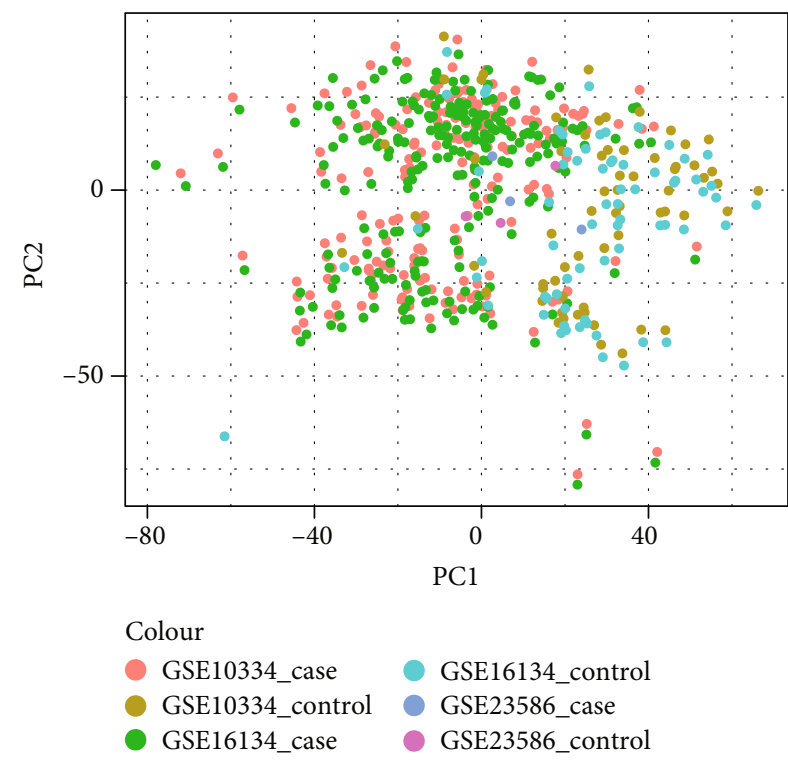

(d)

FIGURe 1: (a, b) PCA analysis results of MDD batches before and after rectification; (c, d) PCA analysis results of PD batch before and after correction.

pairs. In order to further analyze the regulatory effect of significant relationships between crosstalk genes and neuropeptides, the expression values of significant relationships between genes in diseased and healthy groups of MDD and PD were examined, respectively. Subsequently, regression analysis was performed to calculate the differentially regulated link (DRL).

In the data of regression analysis, the regulatory effect of crosstalk genes with neuropeptides was assessed, whereby the expression of crosstalk genes acted as $x$ and expression of neuropeptides as $y$ in the formula $y \sim \beta x+\beta_{0}$.

By calculating this formula, it is possible to obtain the regression coefficients of the crosstalk gene-neuropeptide

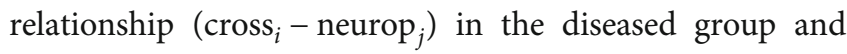
healthy control group of MDD and PD, respectively. Then, the sum of the standard deviations of the relationships in the case group and healthy control group was calculated for both MDD and PD, respectively. The correlation coefficients in all samples of MDD and PD were calculated. Finally, the regulation coefficients of the relationship in differential coexpression genes were calculated according to the formula shown below.

$$
\mathrm{DRL}_{i, j}=\frac{\beta_{\text {case }}-\beta_{\text {control }}}{\sqrt{s d_{\text {case }}^{2}+s d_{\text {control }}^{2}+C C(i, j)_{\text {all }}}} .
$$




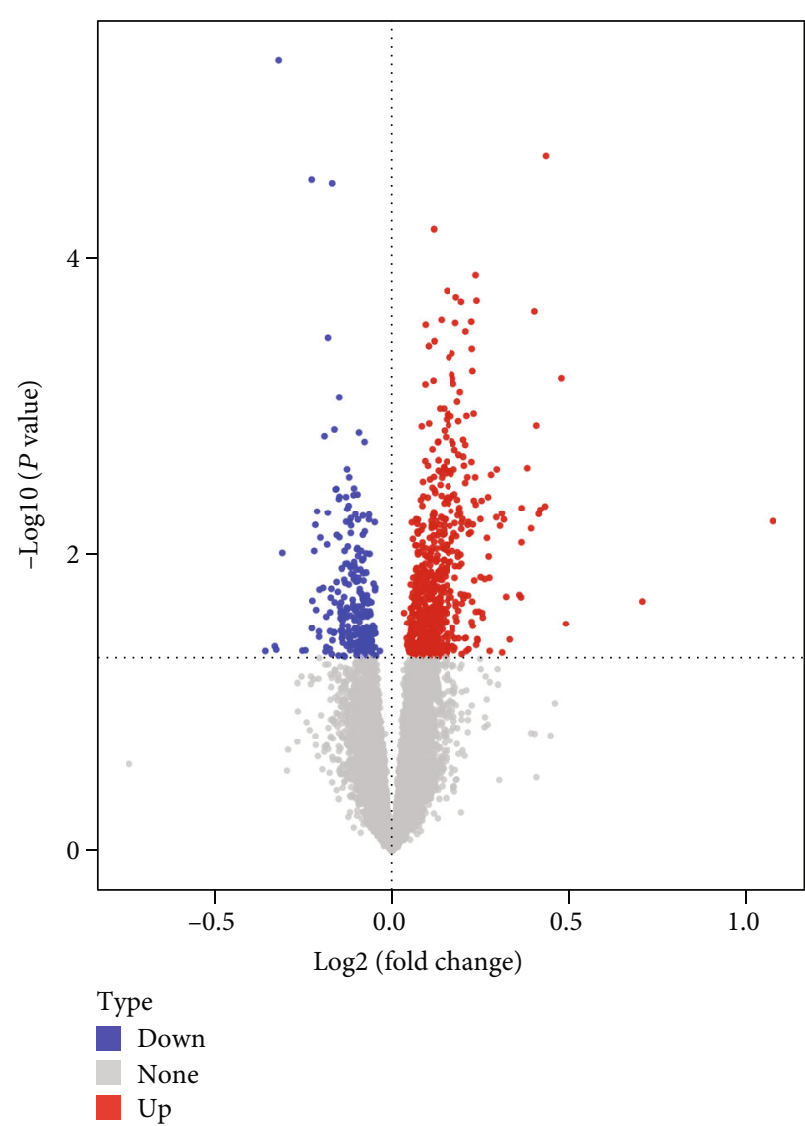

(a)

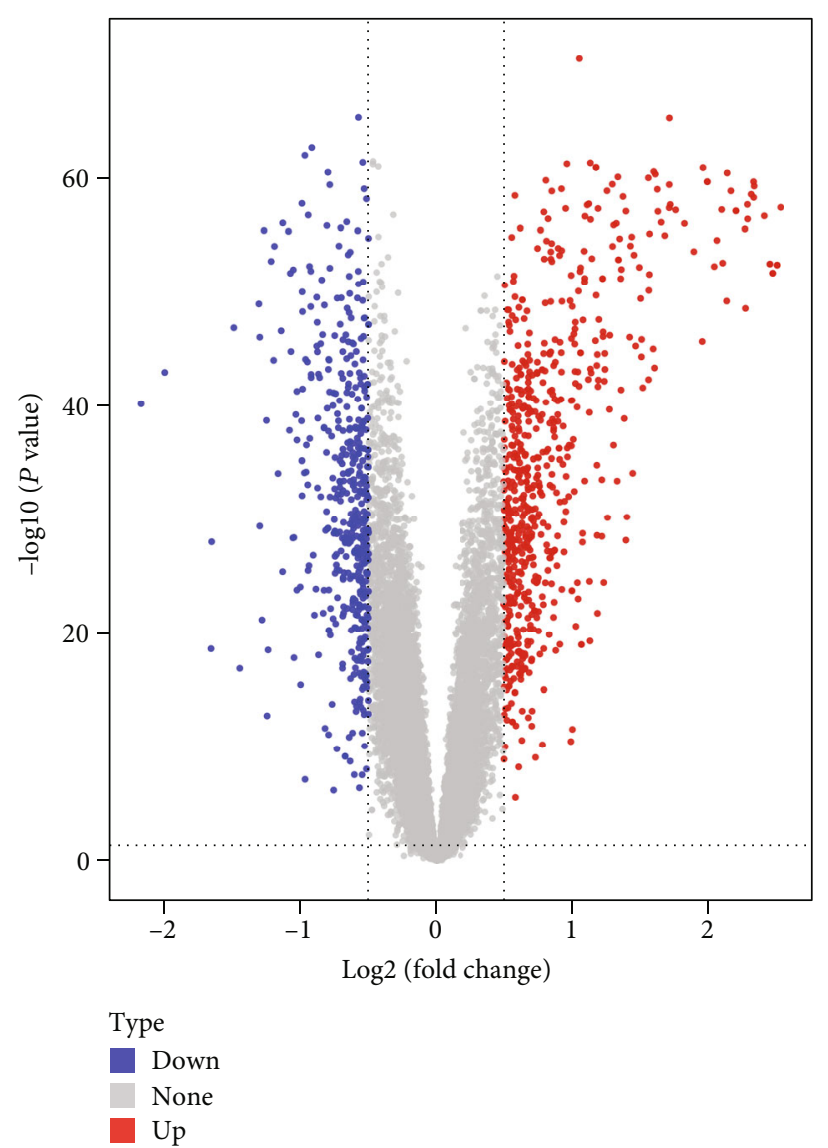

(b)

Figure 2: Volcano maps of differentially expressed genes in (a) MDD and (b) PD.

A positive DRL indicates a consistent regulation of the crosstalk-neuropeptide relationship in case and control samples, while both are either inhibiting or promoting. If the value is negative, the relationship is opposite in case and control regulation modes.

2.6. Functional Analysis of Crosstalk Genes and Neuropeptides. The human pathway-gene pairs were downloaded from KEGG (https://www.kegg.jp/), and the pathways corresponding to crosstalk genes and neuropeptides were extracted. It has been further evaluated whether the pathway contains both crosstalk genes and neuropeptides, followed by an analysis of the pathways regulated by crosstalk genes and neuropeptides. These coacting pathways may be the key pathways for the interaction of the two genes. Cytoscape was used to map the pathways of crosstalk genes and neuropeptides.

2.7. Analysis of Crosstalk Genes and Neuropeptides in the Protein Interaction Network. For experimental verification of protein-protein interactions (PPI), data were downloaded for MINT (http://mint.bio.uniroma2.it/mint/Welcome.do), HPRD (http://www.hprd.org/index_html), BIOGRID (http://thebiogrid.org/), DIP (http://dip.doe-mbi.ucla.edu/ dip/Main.cgi), menthe (http://mentha.uniroma2.it/index .php), PINA (http://cbg.garvan.unsw.edu.au/pina/), Inna-
TABle 2: Statistics of differentially expressed genes.

\begin{tabular}{lccc}
\hline Disease & Up & Down & Total \\
\hline MDD & 674 & 237 & 911 \\
PD & 664 & 461 & 1125 \\
\hline
\end{tabular}

teDB (http://www.innatedb.com/), and Instruct (http:// instruct.yulab.org/index.html). PPI pairs of crosstalk genes and neuropeptides were then extracted. In order to further analyze the relationship between crosstalk genes and neuropeptides in the system biological network, one-step PPI was extended according to the relationship pairs. An indirect pair (cross-other-neuropeptide) that regulated both crosstalk genes and neuropeptides was extracted. Then Cytoscape software was used to construct the PPI network. The relationship links were displayed in the network and the topology properties were analyzed.

2.8. Neuropeptide Screening. The expression values of all neuropeptides in MDD and PD were extracted and the Lasso regression analysis was applied to screen neuropeptides. The expression values of specific neuropeptides in $\mathrm{PD}$ and $\mathrm{MDD}$ were extracted, boxplots were created, and receiver operating curve (ROC) analysis was conducted. In addition, the 

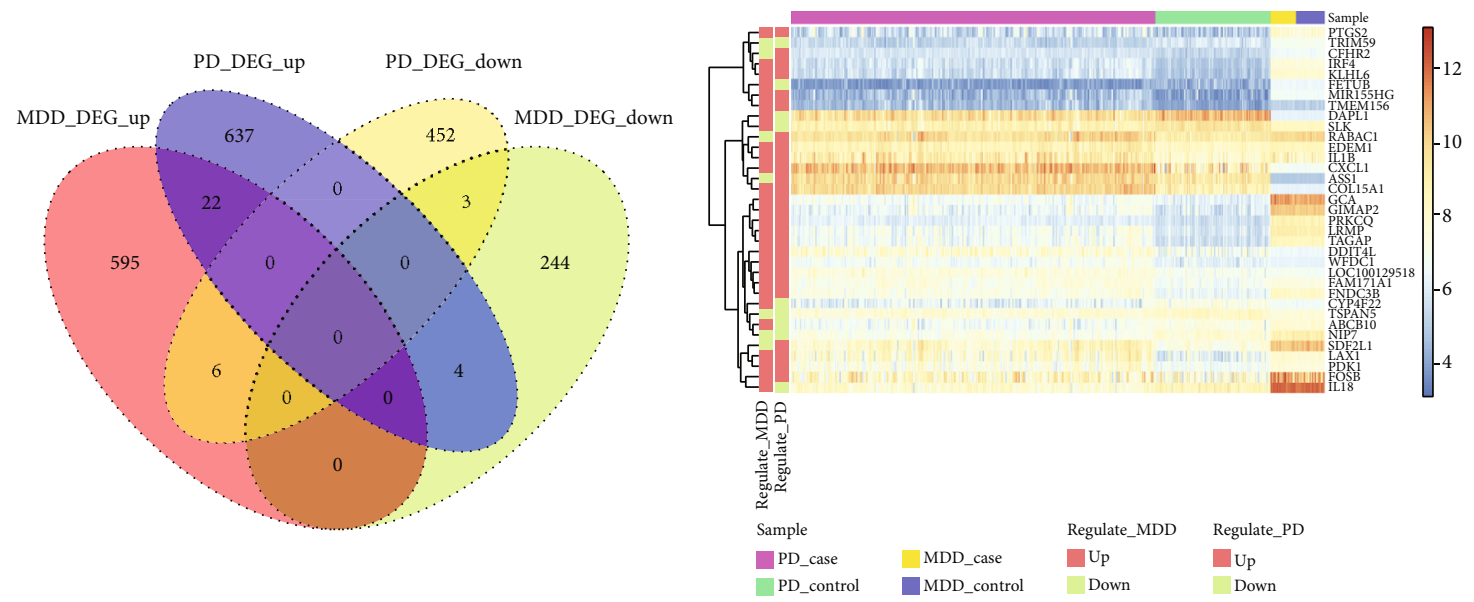

(a)

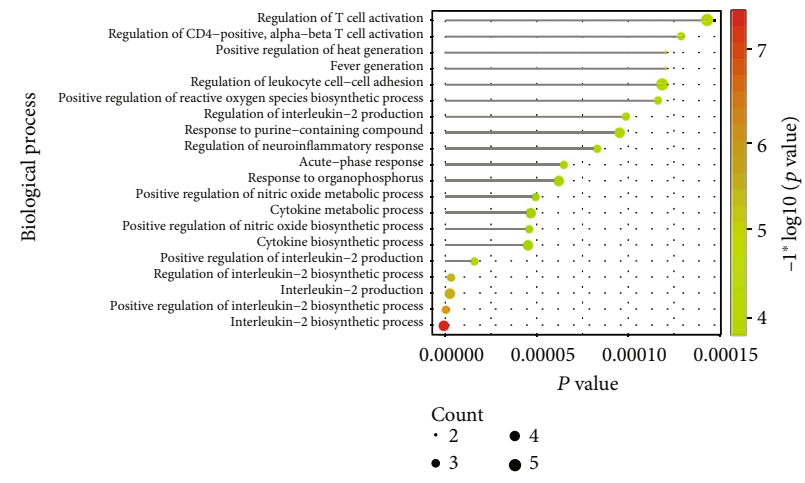

(b)

(c)

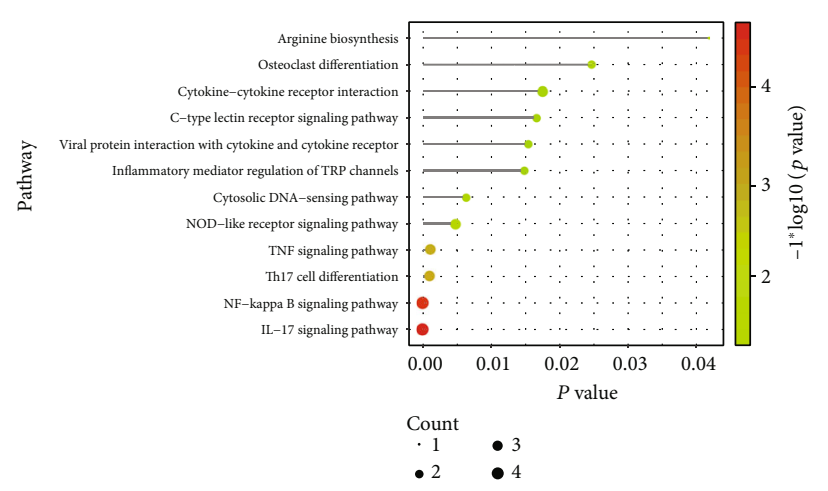

(d)

Figure 3: Regulation function of crosstalk genes. (a) Venn maps of differentially expressed genes obtained from MDD and PD. (b) Heat maps of 35 crosstalk genes in MDD and PD. (c) Top 20 biological processes of significant enrichment of crosstalk genes. (d) All significant KEGG pathways were regulated by significant crosstalk genes.

differential regulatory weights of these specific neuropeptides and their highly correlated crosstalk genes were extracted in MDD and PD. Finally, the interaction pathways for these specific neuropeptides and the corresponding highly correlated crosstalk genes were evaluated, the neuropeptides were associated with the crosstalk genes and the pathways, and the functions influenced by neuropeptides and crosstalk genes were analyzed.

\section{Results}

3.1. Data Preprocessing. After data combination and batch correction, a dataset of MDD and PD was created, wherein MDD contains 30 case and 33 control samples, while PD contains 430 case and 139 control samples. At the same time, PCA analysis results of data before and after correction (Figure 1) for the two diseases were reviewed.

3.2. Differential Expression Analysis. According to the analysis results, the differentially expressed genes were screened and the volcano diagram was used to show the cutoff screening of differentially expressed genes (Figures 2(a) and 2(b)). The number of differential expressed genes obtained is shown in Table 2.
3.3. Crosstalk Gene Screening. The intersection of differentially expressed genes obtained from MDD and PD were the respective crosstalk genes (Figure 3(a)). A total of 35 crosstalk genes were obtained (Figure 3(b)). clusterProfiler package of $\mathrm{R}$ language was used for functional enrichment analysis of these 35 crosstalk genes (significance level $p$ value $<0.05$ ). Figure $3(\mathrm{c})$ shows the biological processes in which the 35 crosstalk genes were mainly involved (Figure 3(c)). The 35 crosstalk genes mainly regulated the IL-17 signaling pathway, NF-Kappa B signaling pathway, and TNF signaling pathway (Figure 3(d)).

3.4. The Links between Crosstalk Genes and Neuropeptides. A total of 102 neuropeptides were extracted from the database, and the expression values of these genes in MDD and PD were further extracted to construct the heat map (Figure 4). The results showed that neuropeptides were not differentially expressed in MDD and PD. Moreover, crosstalk genes and neuropeptides had no intersection, indicating that no gene was revealed to be both crosstalk gene and neuropeptide.

In order to further analyze the role of crosstalk genes and neuropeptides in the entire biological network, the relationship pairs of the direct interaction between crosstalk genes and neuropeptides were extracted according to the known 


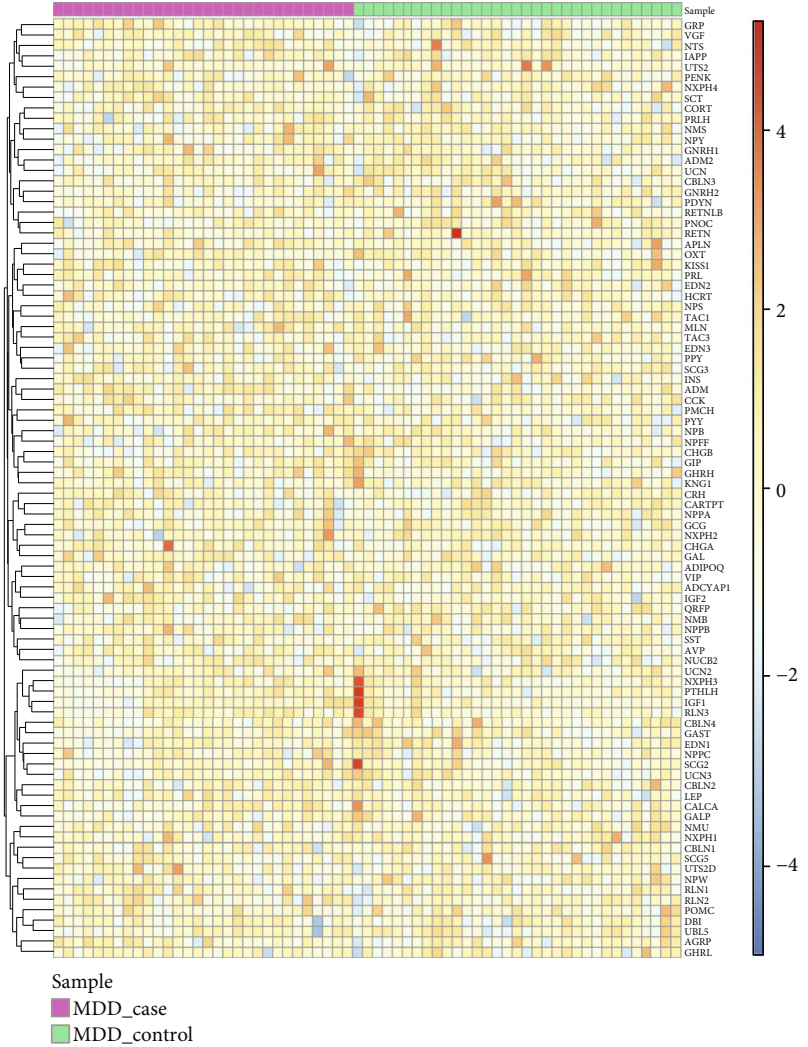

(a)

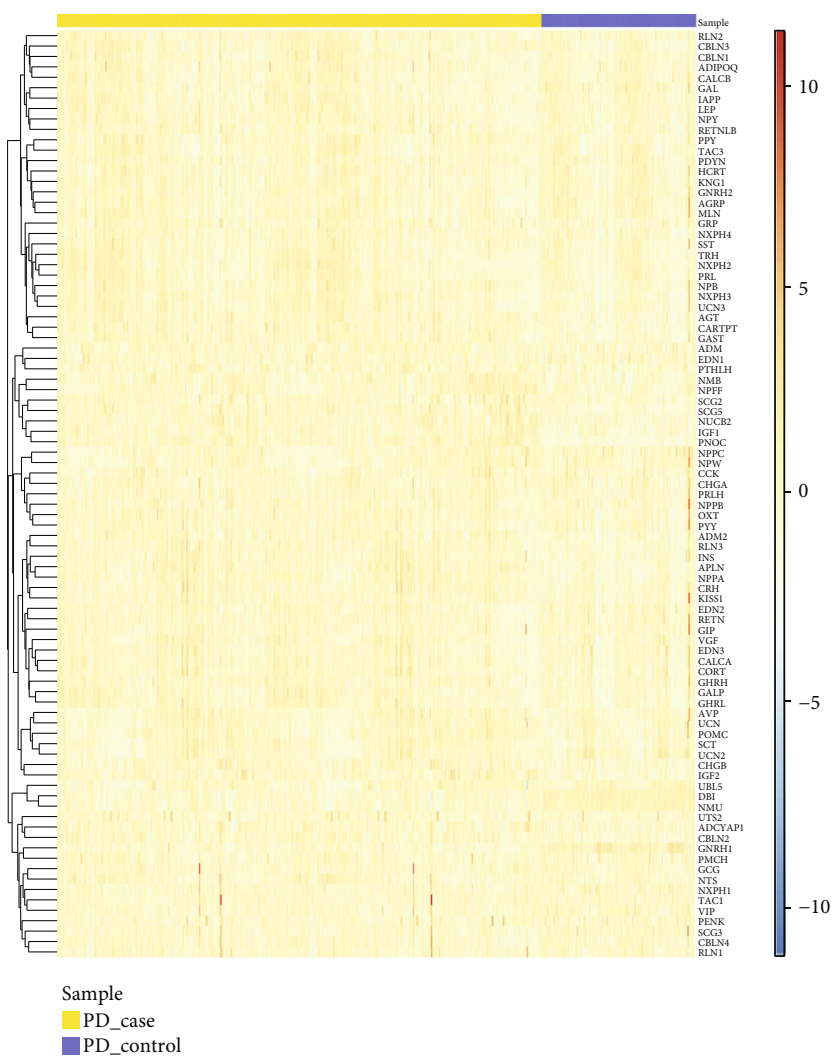

(b)

Figure 4: Heat maps of neuropeptide expression in (a) MDD and (b) PD.

PPI relationship pairs. As a result, no direct interaction pairs were obtained. According to the interaction proteins of crosstalk genes, a step extension and subsequent screening of the extended proteins as genes of neuropeptides obtained a total of 164 relationship pairs. Then Cytoscape software was used to construct the PPI network (Figure 5).

Topological properties of the top 20 genes were screened out according to the degree in descending order. The results are shown in Table 3.

From the PPI network, neuropeptide genes NUCB2, DBI, and UBL5 could interact with more genes, thus indirectly interacting with crosstalk genes.

\subsection{Differential Coexpression Regulation Links between} Crosstalk Genes and Neuropeptides. To identify the function of neuropeptides in MDD and PD, the correlation between crosstalk genes and neuropeptides in MDD and PD was assessed firstly. Figure 6 shows the correlation between crosstalk genes and neuropeptides in the case group of MDD and PD (Figure 6).

The significant correlation pairs in the case and control groups of MDD and PD were selected, and the regulatory relationships of differential coexpression were analyzed. Finally, a total of 181 significant relationship pairs were obtained. The pathways jointly regulated by crosstalk genes and neuropeptides in a significant pair were obtained, and the crosstalk gene-pathway-neuropeptide network was con- structed using Cytoscape software (Figure 6(c)). The crosstalk gene-pathway-neuropeptide network showed that the crosstalk genes between MDD and PD could act with neuropeptides indirectly.

3.6. Screening and Analysis of Neuropeptides. The expression profiles of 102 neuropeptides in MDD and PD were obtained and the specific neuropeptides were screened by lasso regression (Figure 7).

Through lasso regression, 9 neuropeptides in MDD and 43 neuropeptides in PD were obtained. Four intersected genes were obtained, i.e., ADM, IGF2, PDYN, and RETN. The expression values of these four genes in MDD and $\mathrm{PD}$ were extracted, and ROC analysis of single genes was performed (Figures 7(e) and 7(f)) to check the accuracy of the expression values of these genes at the expression level. The results showed that IGF2 was highly predictive in MDD and PD. ADM was better than the other three genes in predicting MDD disease.

According to the differential coexpression regulatory relationship, the DRL of highly correlated coexpression relationships between crosstalk genes and neuropeptides was obtained (Figure 8(a)). Then, the crosstalk genes were analyzed together with these four neuropeptide genes (Figure $8(\mathrm{~b})$ ). A total of 13 crosstalk genes were differentially coexpressed with four neuropeptides. The expression profiles of these 13 genes in MDD and PD were extracted, and 


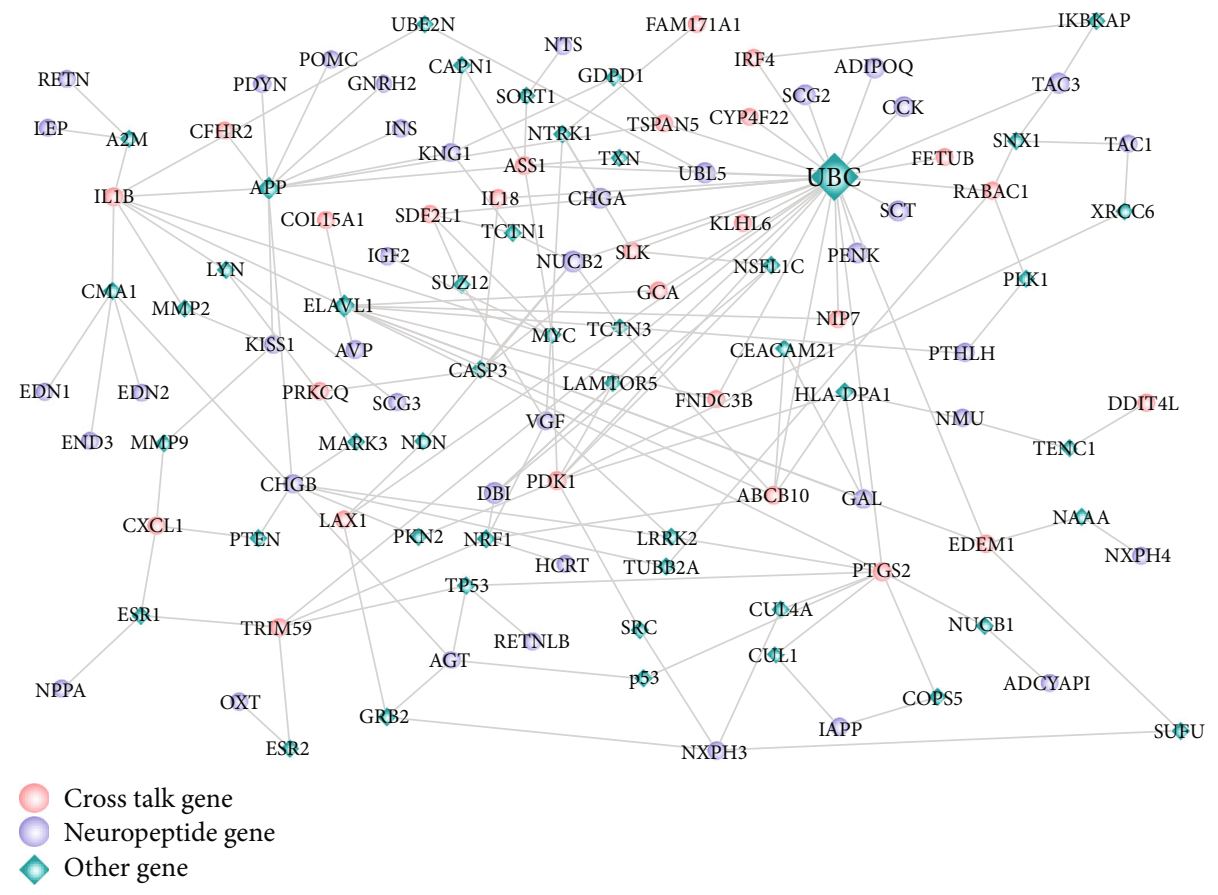

FIGURE 5: Crosstalk genes and neuropeptides in the PPI-network. In the network, crosstalk genes and neuropeptides are linked by bridging genes.

TABLE 3: Topological properties of top 20 genes.

\begin{tabular}{|c|c|c|c|c|c|c|}
\hline Name & Label & Degree & Average shortest path length & Betweenness centrality & Closeness centrality & Topological coefficient \\
\hline UBC & & 418 & 2.339286 & 0.553074 & 0.427481 & 0.056261 \\
\hline APP & & 64 & 3.125 & 0.160397 & 0.32 & 0.114833 \\
\hline ELAVL1 & & 64 & 2.946429 & 0.157445 & 0.339394 & 0.119835 \\
\hline NUCB2 & Neuropeptide & 50 & 3.169643 & 0.032524 & 0.315493 & 0.233333 \\
\hline DBI & Neuropeptide & 48 & 3.241071 & 0.017919 & 0.30854 & 0.291667 \\
\hline UBL5 & Neuropeptide & 42 & 3.098214 & 0.018099 & 0.322767 & 0.344828 \\
\hline TAC3 & Neuropeptide & 42 & 3.276786 & 0.020327 & 0.305177 & 0.356322 \\
\hline CHGA & Neuropeptide & 42 & 3.276786 & 0.013341 & 0.305177 & 0.516667 \\
\hline SCT & Neuropeptide & 38 & 3.330357 & 0 & 0.300268 & 0 \\
\hline SCG2 & Neuropeptide & 38 & 3.330357 & 0 & 0.300268 & 0 \\
\hline PENK & Neuropeptide & 38 & 3.330357 & 0 & 0.300268 & 0 \\
\hline CCK & Neuropeptide & 38 & 3.330357 & 0 & 0.300268 & 0 \\
\hline ADIPOQ & Neuropeptide & 38 & 3.330357 & 0 & 0.300268 & 0 \\
\hline GAL & Neuropeptide & 22 & 3.723214 & 0.011257 & 0.268585 & 0.388889 \\
\hline PTGS2 & Crosstalk & 18 & 2.758929 & 0.16726 & 0.36246 & 0.133333 \\
\hline PTHLH & Neuropeptide & 18 & 3.848214 & 0.005528 & 0.259861 & 0.5 \\
\hline CHGB & Neuropeptide & 18 & 3.401786 & 0.060159 & 0.293963 & 0.166667 \\
\hline PDK1 & Crosstalk & 16 & 2.830357 & 0.115809 & 0.353312 & 0.146429 \\
\hline IL1B & Crosstalk & 16 & 3.0625 & 0.190297 & 0.326531 & 0.132813 \\
\hline MYC & & 16 & 3.160714 & 0.0628 & 0.316384 & 0.231579 \\
\hline
\end{tabular}

the expressions of these crosstalk genes in MDD and PD were analyzed (Figures 8(c) and 8(d)), showing that FOSB was highly expressed in MDD and PD.

In addition, pathways related to ADM, IGF2, PDYN, and RETN were extracted and pathways shared by neuro- peptides and crosstalk genes were analyzed based on pathway genes (Figure 9).

Figure 8 (b) shows that ADM and RETN are differentially coexpressed with multiple crosstalk genes. In the functional analysis, ADM was mainly involved in neuroactive ligand- 


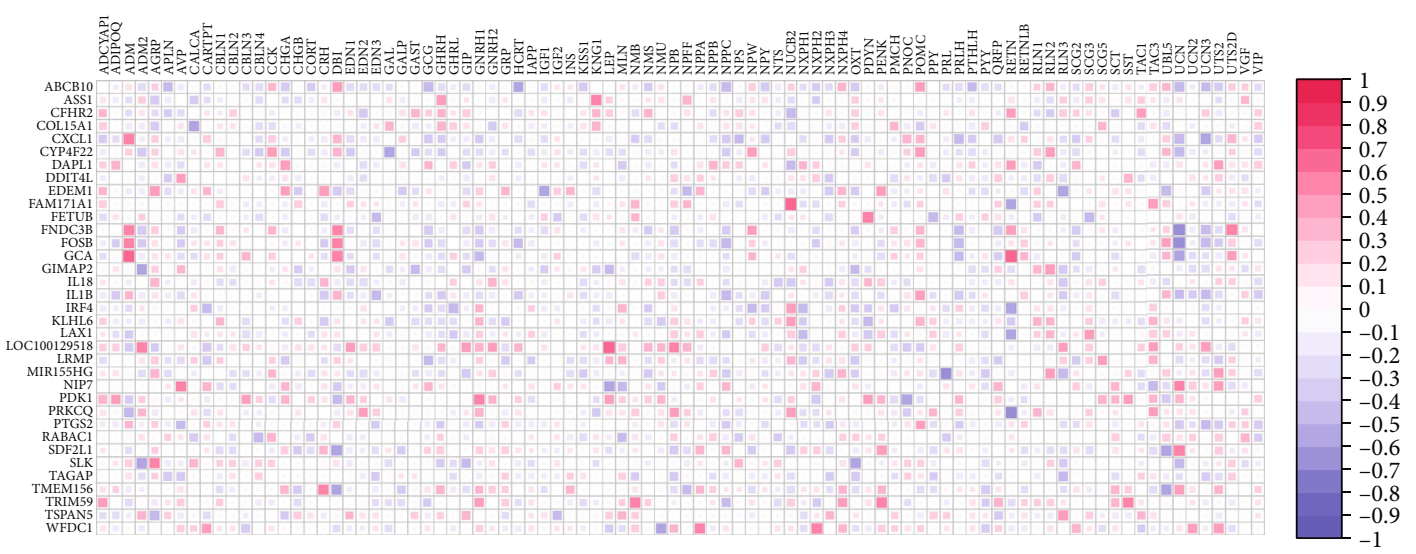

(a)

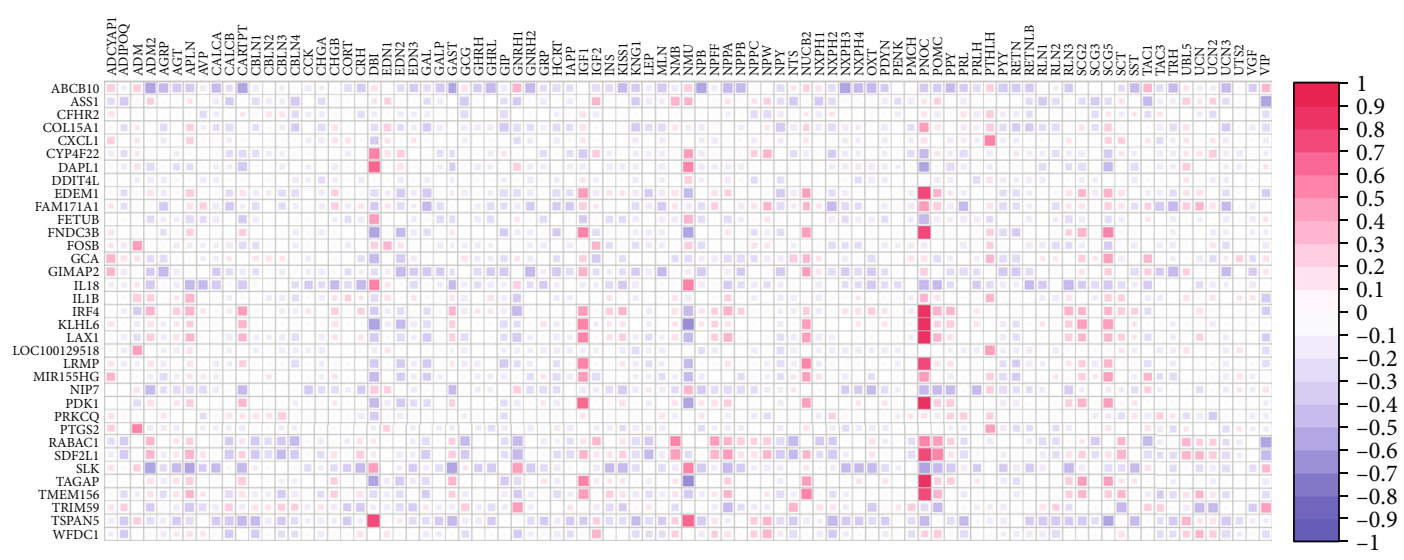

(b)

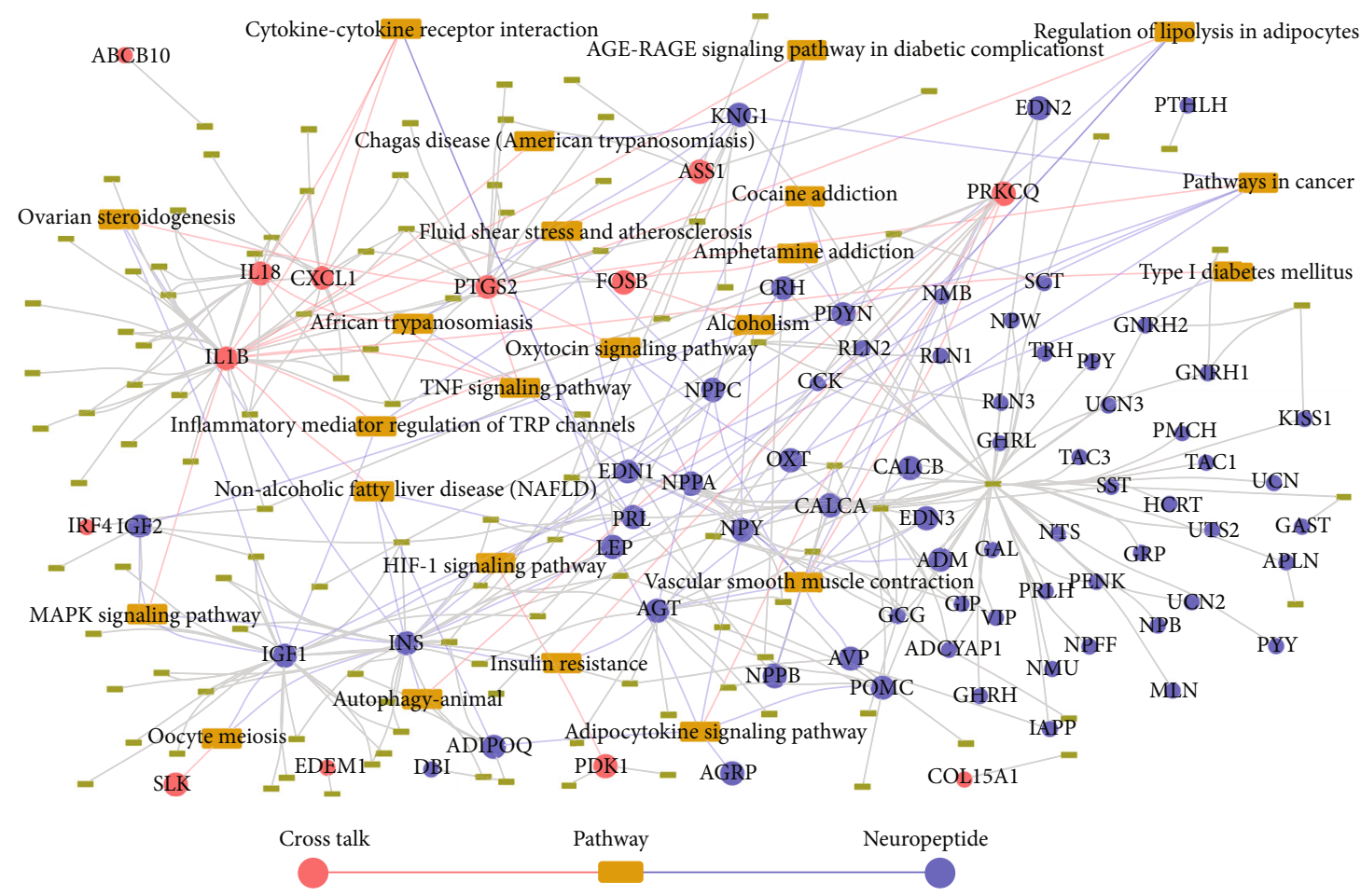

(c)

FIGURE 6: Correlation between crosstalk genes and neuropeptides in disease groups of (a) MDD and (b) PD and (c) crosstalk gene-pathwayneuropeptide network. 


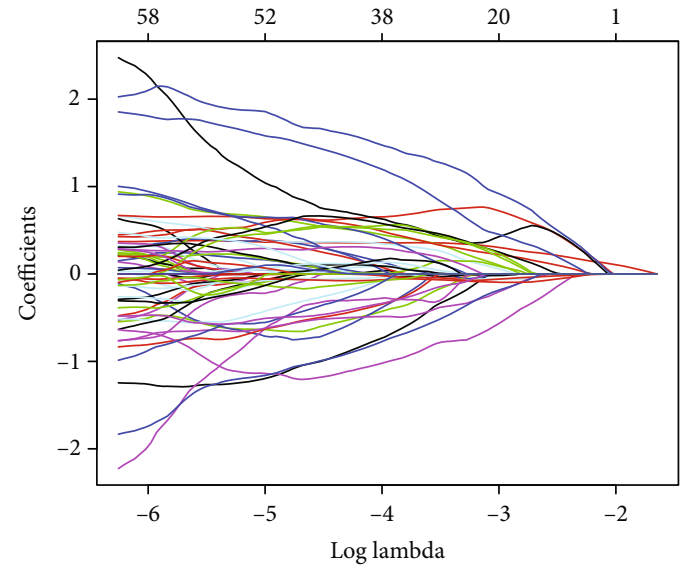

(a)

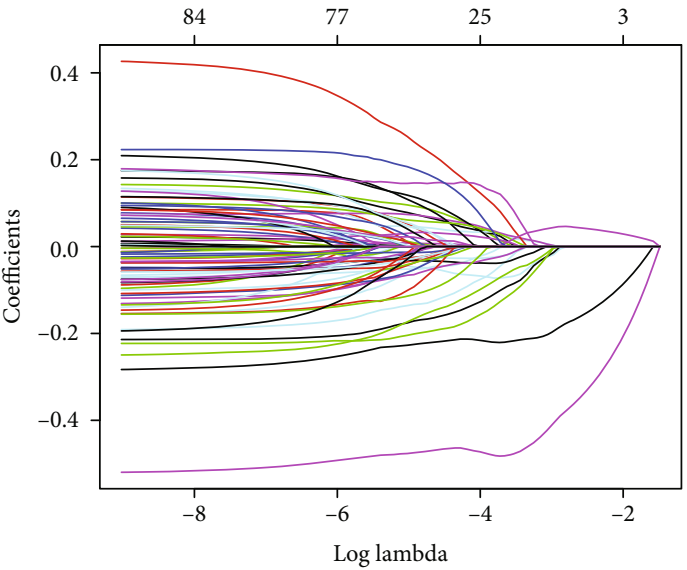

(c)

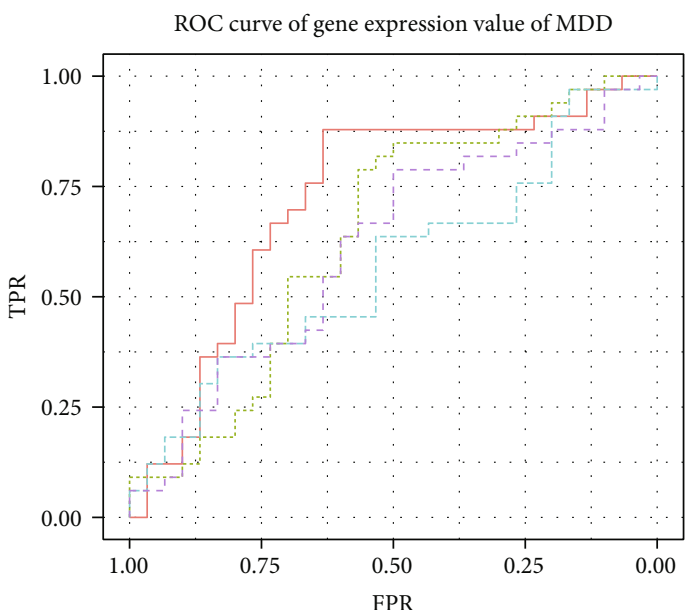

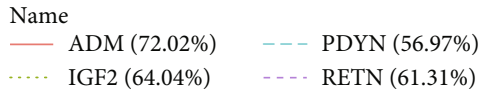

(e)

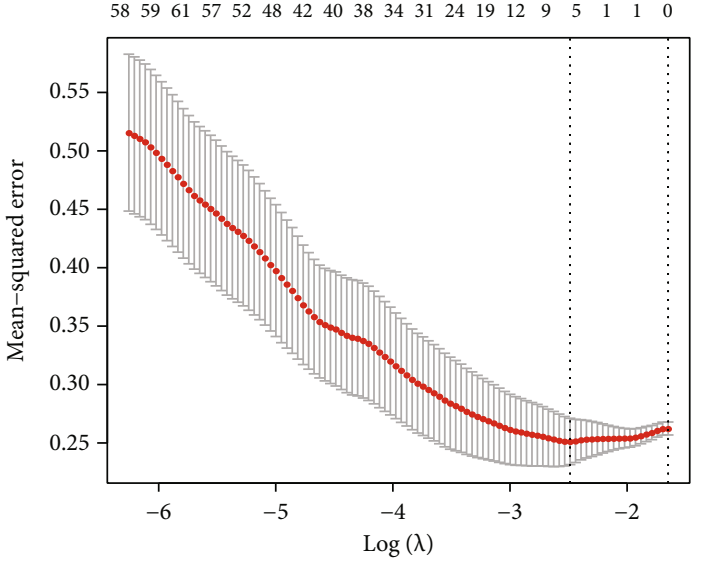

(b)

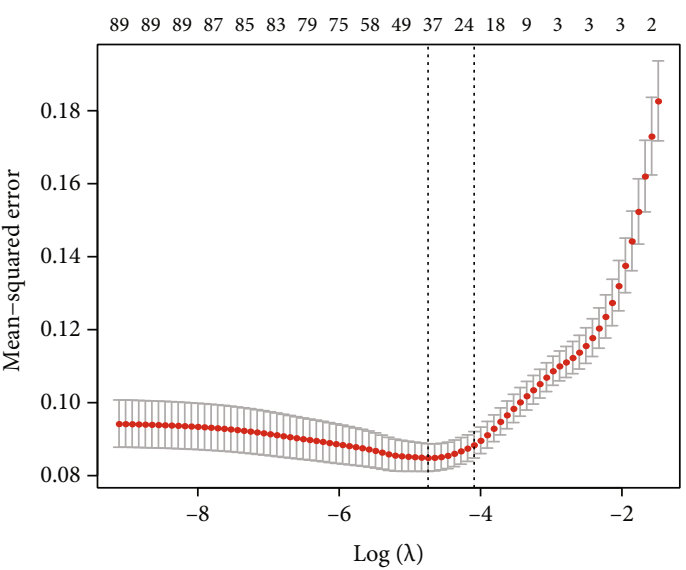

(d)

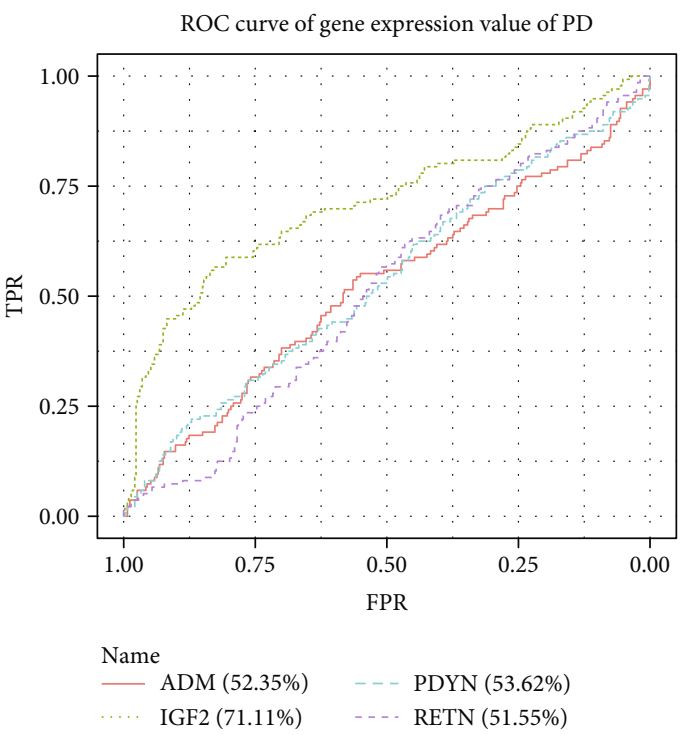

(f)

FIGURE 7: Lasso regression, modeling results, and CV search for the best lambda value. (a) Modeling results of neuropeptides in MDD Lasso regression analysis. The abscissa is log (lambda), and the ordinate corresponds to the correlation coefficient of the modeling process. (b) Neuropeptide screening the relationship between lambda value and mean square error in MDD Lasso regression analysis. The abscissa is $\log$ (lambda) and the ordinate is mean square error. There are two dashed lines in the figure, one is the value of $\lambda$ with the minimum mean square error and the other is the value of $\lambda$ with the standard error from the minimum mean square error. (c) Modeling results of neuropeptides in PD Lasso regression analysis; (d) neuropeptide screening lambda value and mean square error in PD Lasso regression analysis. (e) ROC results of ADM, IGF2, PDYN, and RETN in MDD; (f) ROC results of ADM, IGF2, PDYN, and RETN in PD. 


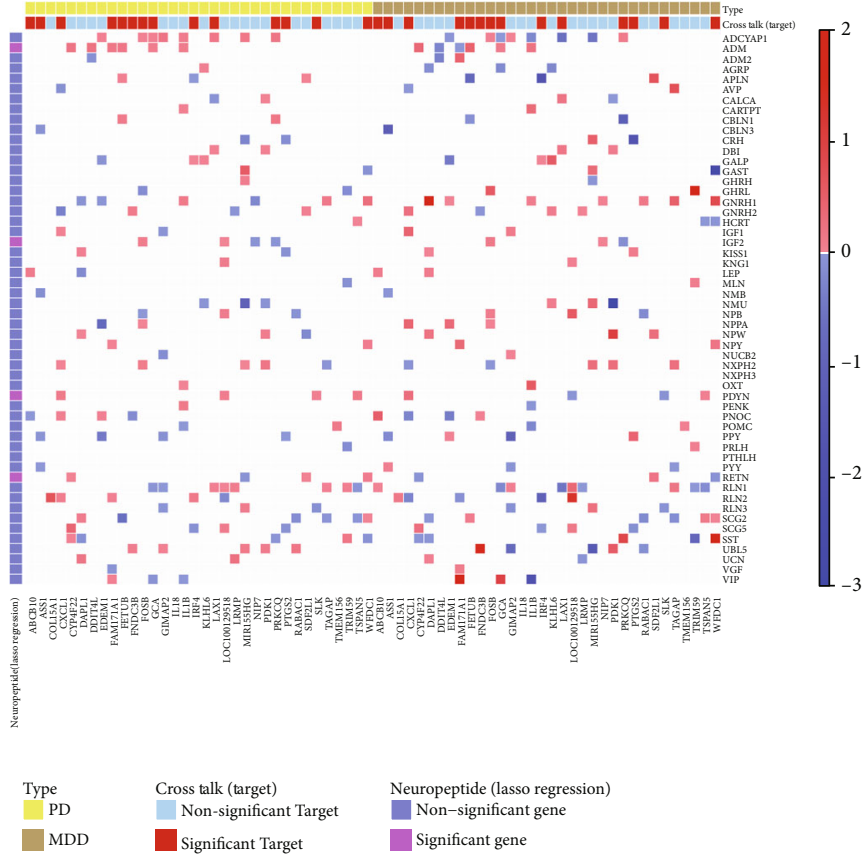

(a)

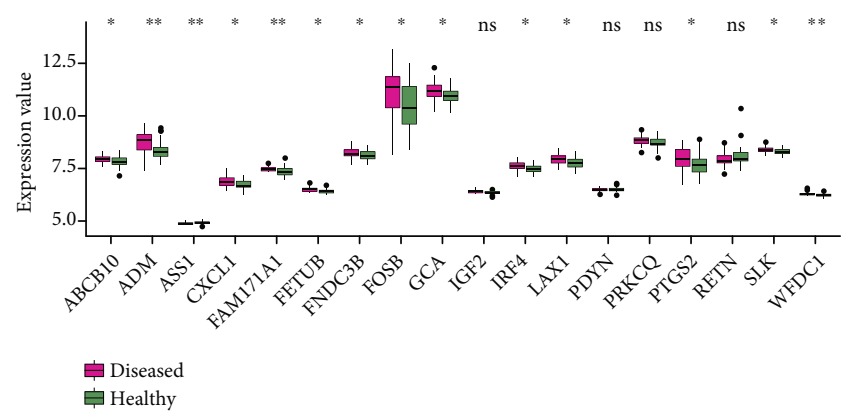

(c)

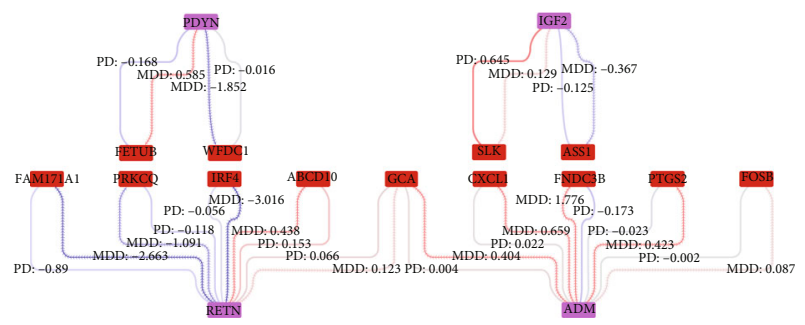

(b)

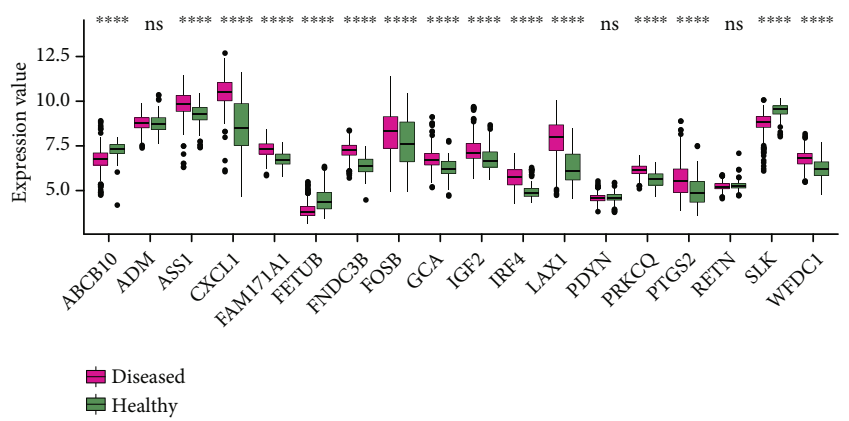

(d)

FIGURE 8: Analysis of crosstalk genes differentially coexpressed with specific neuropeptides. (a) Heat map of highly correlated differential coexpression regulation of crosstalk genes and neuropeptides. (b) Pairs of differential coexpression strength of crosstalk genes differentially coexpressed with specific neuropeptides. The thicker the line, the greater the intensity of regulation; the value on the line represents the specific intensity of regulation; the positive value indicates that the relationship has a consistent trend of regulation in diseases and normal tissues; the negative value indicates inconsistent trend of regulation. (c) Expression of crosstalk genes, which were differentially coexpressed with specific neuropeptides in MDD. In the figure, the relationship between $p$ and * is as follows: ns indicates $p>0.05, *$ represents $p \leq 0.05, * *$ represents $p \leq 0.01, * * *$ represents $p \leq 0.001, * * * *$ means $p \leq 0.0001$. (d) Expression of crosstalk genes, which were differentially coexpressed with specific neuropeptides in PD.

receptor interaction and vascular smooth muscle contraction. ADM indirectly interacts with FOSB through the neuroactive ligand-receptor interaction pathway, thus affecting the abnormalities of biological processes. ADM and FOSB were differentially coexpressed at the gene expression level and had opposite regulation patterns on MDD and PD in general. In addition, FOSB also regulates the IL-17 signaling pathway. IGF2 regulated the MAPK signaling pathway, PI3K-Akt signaling pathway, and Ras signaling pathway. In the differential coexpression analysis, it was found that IGF2 and ASS1 were highly coexpressed and the regulatory trends were inconsistent between MDD-diseased and healthy control groups as well as PD-diseased and healthy control groups. From the function diagram, it can be seen that
ADM, IGF2, PDYN, and RETN can indirectly affect the potential biological function between MDD and PD disease through the function of multiple crosstalk genes.

\section{Discussion}

The neuropeptide genes ADM, IGF2, PDYN, and RETN were found to be intersected between PD and MDD. FOSB was significantly coexpressed with these neuropeptides. Relevant pathways for these genes were the IL-17 signaling pathway, MAPK signaling pathway, $\mathrm{Pi} 3 \mathrm{~K}-\mathrm{Akt}$ signaling pathway, Ras signaling pathway, Neuroactive Ligand-receptor interaction, and vascular smooth muscle contraction. 


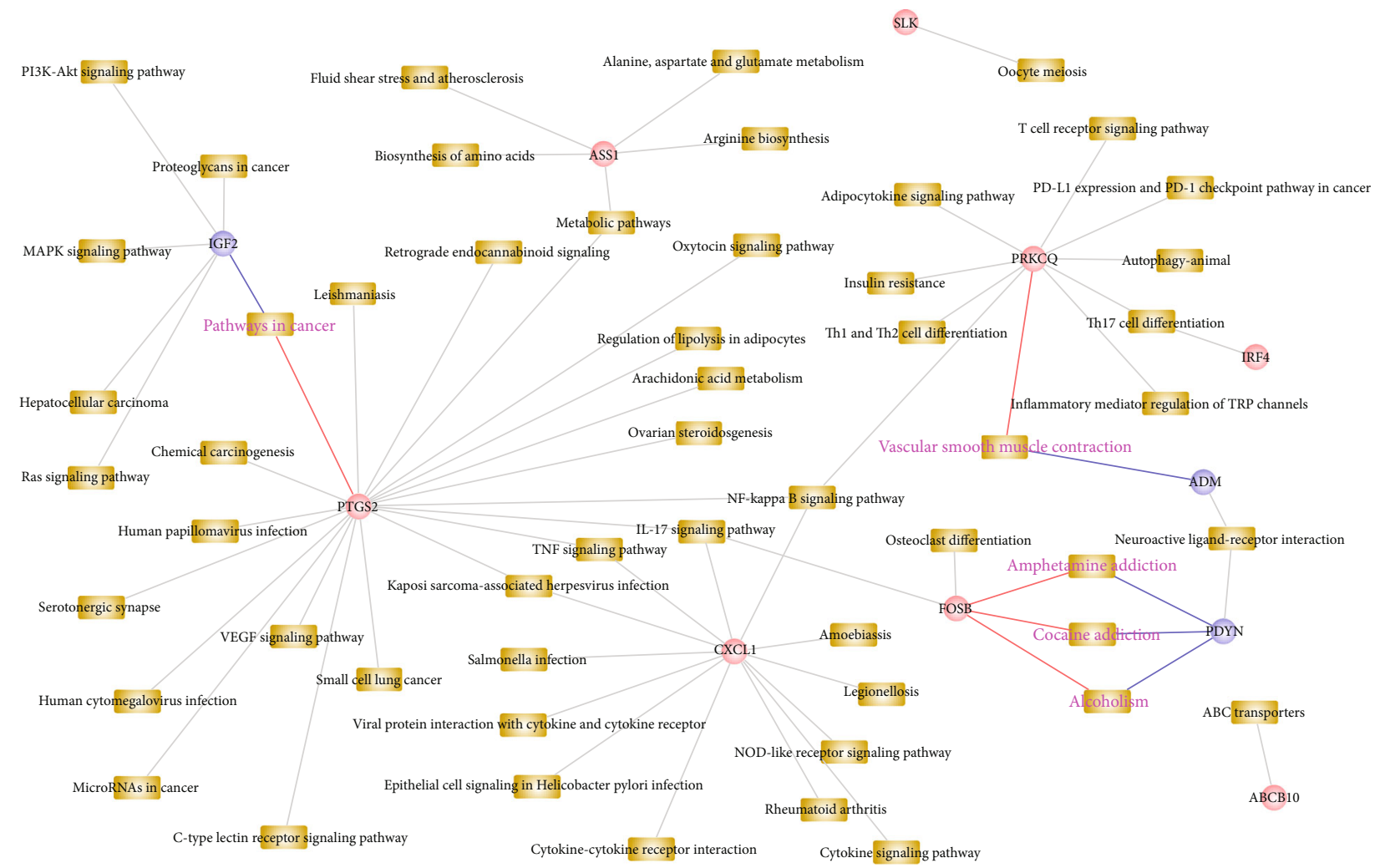

FIGURE 9: Pathways involved in crosstalk genes differentially coexpressed with ADM, IGF2, PDYN, and RETN. The blue dots represent the four neuropeptides, the pink dots represent crosstalk genes, and the yellow box represents the pathways that genes are associated with.

This is the first bioinformatics study, investigating the crosstalk genes and related neuropeptides between PD and MDD. A relationship between $\mathrm{PD}$ and MDD has been extensively discussed in literature, whereby a relationship between these two diseases appear probable [6, 9-12]. The causal link between those two diseases was supposed to be within PD-induced systemic inflammation, leading to a neuroinflammation due to the expression of proinflammatory cytokines $[13,14]$. This current study revealed several neuropeptide genes on the transcriptomic level, which will be discussed in the following.

Adrenomedullin (ADM) is a peptide hormone with important roles in the regulation of the cardiovascular and lymphatic systems [17]. ADM was revealed to be a potential biomarker and candidate for therapeutic interventions [17]. A study investigating tissue punches from dentate gyrus revealed $\mathrm{ADM}$ as an inhibitor of angiogenesis to be related to neuroinflammation in patients with MDD [18]. Another study revealed that increased levels of ADM and $\mathrm{NO}$ in serum of patients would be associated to MDD and related psychomotor retardation [19]. The ADM-NO axis was also elevated due to periodontal pathogenic bacteria, especially Aggregatibacter actinomycetemcomitans [20]. Similarly, another study found the ADM-NO-axis to be a functional linkage to PD severity [21]. A lack of sensitivity to ADM could also be related to the bacterial invasion of Porphyromonas gingivalis, another periodontal pathogenic bacterium [22]. Furthermore, ADM was found to affect the therapeutic efficiency of the antidepressant paroxetine [23].
Altogether, ADM and the ADM-NO-axis support the role of neuroinflammation as well as the potential relevance of oral pathogens in the relationship between PD and MDD.

Insulin-like growth factor 2 (IGF2) is a hormone regulating cell proliferation, migration, differentiation, and survival [24]. IGF2 was found to mediate depressive behaviors in the brain of rats [25]. Variable methylation of IGF2 was found to be related to the clinical manifestation of MDD in monozygotic twins [26]. Additionally, IGF2 binds on insulin receptor in the brain, whereby dysregulation of IGF2 leads to neuropathological processes [27]. Alongside with IGF1, IGF2 was found to be associated to neuroinflammatory processes [28]. In this respect, insulin-like growth factor 2 mRNA-binding protein 1 was found to promote the NF- $\kappa \mathrm{B}$ signaling way, what is induced by lipopolysaccharides, which are important virulence factors of periodontal pathogenic bacteria [29]. This supports the hypothesis of the role of $\mathrm{PD}$ in inducing neuroinflammation related to MDD.

Prodynorphin (PDYN) is an endogenous agonist of the $\mathrm{k}$-opioid receptors, having modulatory effects related to addiction; the expression of this peptide is altered in the brain of patients with mental disorders $[30,31]$. It has been shown that an impairment of PDYN in the amygdala is associated with MDD [32]. Until now, there is no study reporting the potential relevance of PYDN in $\mathrm{PD}$, making a discussion of this issue difficult.

Resistin (RETN) is a peptide that is secreted by adipocytes, playing roles in metabolism [33]. As an adipokine, RETN is well known to play an important role in 
development of MDD, while a recent meta-analysis showed that the serum level of resistin was lower in individuals with MDD compared to healthy participants [34]. In patients with MDD, RETN was found to be associated to free cortisol concentrations and therapy outcome [35]. Thereby, a link between obesity and MDD was reported [36]. Moreover, RETN is involved in the interrelationship between MDD and diabetes, whereby neuroinflammation was reported to be of certain relevance [37]. This is a potential and interesting connecting point of MDD and PD; RETN was found to be elevated in gingival crevicular fluid and serum of patients with $\mathrm{PD}$, although this was not related to systemic inflammatory diseases [38]. Another review article showed RETN to be a potential biomarker in the interrelationship between $\mathrm{PD}$ and diabetes [39]. In this axis, a causal relation between MDD and PD can be supported.

Lastly, FOSB was significantly coexpressed with these neuropeptides. FOSB is a member of the Fos family of transcription factors and thereby a regulator of stress and antidepressant response [40]. The induction of FOSB in the hippocampus was reported to be critical in addiction and MDD [41]. A network analysis showed that FOSB was one hub gene for depressed suicide [42]. For PD, an analysis based one gene expression data found FOSB to be an inflammationrelated gene that might be involved in the development and progression of PD [43]. Accordingly, an influence of this gene on the relation between PD and MDD appears conceivable. Altogether, the hypothesis of neuroinflammation as well as the effect of cofactors like diabetes and obesity in the interplay between PD and MDD can be supported by the bioinformatics data within the current study. Accordingly, interdisciplinary therapeutic and preventive approaches would be needed to comprehensively manage the complex problem of patients suffering from those diseases.

This is the first bioinformatics study investigating the crosstalk and related neuropeptides between PD and MDD. The methodology was comprehensive and revealed a variety of results. However, the bioinformatics approach has several limitations, especially the missing validation of the findings. This must be recognized in the interpretation of the findings. Moreover, there are no data available regarding the included patients. Different patients with MDD and PD were included in this analysis, and thereby, the cohort could be very heterogeneous. Based on these limitations, clinical studies, which evaluate the findings on patients suffering from MDD and $\mathrm{PD}$, are needed. As long as these findings are not available, the results and respective conclusions of this bioinformatics study remain speculative. Thereby, it must be recognized that all findings in the current study were only revealed on the transcriptomic level.

\section{Conclusion}

The neuropeptide genes ADM, IGF2, PDYN, and RETN were found to be intersected between PD and MDD, and FOSB was a crosstalk gene, which was related to these neuropeptides on the transcriptomic level. These findings could be a basis for future research in the field, needing further validation.

\section{Data Availability}

The datasets used and/or analyzed during the current study are available from the corresponding author upon reasonable request.

\section{Conflicts of Interest}

The authors declare that they have no competing interests.

\section{References}

[1] A. J. Ferrari, A. J. Somerville, A. J. Baxter et al., "Global variation in the prevalence and incidence of major depressive disorder: a systematic review of the epidemiological literature," Psychological medicine, vol. 43, no. 3, pp. 471-481, 2013.

[2] S. Moussavi, S. Chatterji, E. Verdes, A. Tandon, V. Patel, and B. Ustun, "Depression, chronic diseases, and decrements in health: results from the World Health Surveys," The Lancet, vol. 370, no. 9590, pp. 851-858, 2007.

[3] M. Kennis, L. Gerritsen, M. van Dalen, A. Williams, P. Cuijpers, and C. Bockting, "Prospective biomarkers of major depressive disorder: a systematic review and meta-analysis," Molecular psychiatry, vol. 25, no. 2, pp. 321-338, 2020.

[4] V. Krishnan and E. J. Nestler, "Linking molecules to mood: new insight into the biology of depression," The American Journal of Psychiatry, vol. 167, no. 11, pp. 1305-1320, 2010.

[5] R. Mayeux, "Biomarkers: potential uses and limitations," NeuroRx, vol. 1, no. 2, pp. 182-188, 2004.

[6] M. G. Cademartori, M. T. Gastal, G. G. Nascimento, F. F. Demarco, and M. B. Corrêa, "Is depression associated with oral health outcomes in adults and elders? A systematic review and meta-analysis," Clinical Oral Investigations, vol. 22, no. 8, pp. 2685-2702, 2018.

[7] D. F. Kinane, P. G. Stathopoulou, and P. N. Papapanou, "Periodontal diseases," Nature Reviews Disease Primers, vol. 3, no. $1,2017$.

[8] N. J. Kassebaum, E. Bernabé, M. Dahiya, B. Bhandari, C. J. Murray, and W. Marcenes, "Global burden of severe periodontitis in 1990-2010," Journal of Dental Research, vol. 93, no. 11, pp. 1045-1053, 2014.

[9] M. M. Araújo, C. C. Martins, L. C. Costa et al., "Association between depression and periodontitis: a systematic review and meta-analysis," Journal of Clinical Periodontology, vol. 43, no. 3, pp. 216-228, 2016.

[10] D. X. Zheng, X. N. Kang, Y. X. Wang et al., "Periodontal disease and emotional disorders: a meta-analysis," Journal of Clinical Periodontology, vol. 48, no. 2, pp. 180-204, 2021.

[11] G. G. Nascimento, M. T. Gastal, F. R. M. Leite et al., "Is there an association between depression and periodontitis? A birth cohort study," Journal of Clinical Periodontology, vol. 46, no. 1, pp. 31-39, 2018.

[12] C. C. Hsu, Y. C. Hsu, H. J. Chen et al., "Association of periodontitis and subsequent Depression," Medicine, vol. 94, no. 51, p. e2347, 2015.

[13] S. Hashioka, K. Inoue, T. Miyaoka et al., "The possible causal link of periodontitis to neuropsychiatric disorders: more than psychosocial mechanisms," International Journal of Molecular Sciences, vol. 20, no. 15, p. 3723, 2019.

[14] S. Hashioka, K. Inoue, M. Hayashida, R. Wake, A. Oh-Nishi, and T. Miyaoka, "Implications of systemic inflammation and 
periodontitis for major depression," Frontiers in Neuroscience, vol. 12, no. 12, 2018.

[15] M. Martínez, D. Martín-Hernández, L. Virto et al., "Periodontal diseases and depression: a pre-clinical in vivo study," Journal of Clinical Periodontology, vol. 48, no. 4, pp. 503-527, 2021.

[16] J. Jin, M. Guang, A. C. Ogbuehi et al., "Shared molecular mechanisms between Alzheimer's disease and periodontitis revealed by transcriptomic analysis," BioMed Research International, vol. 2021, Article ID 6633563, 22 pages, 2021.

[17] J. P. Fischer, S. Els-Heindl, and A. G. Beck-Sickinger, "Adrenomedullin - Current perspective on a peptide hormone with significant therapeutic potential," Peptides, vol. 131, p. 170347, 2020.

[18] G. J. Mahajan, E. J. Vallender, M. R. Garrett et al., “Altered neuro-inflammatory gene expression in hippocampus in major depressive disorder," Progress in Neuro-Psychopharmacology \& Biological Psychiatry, vol. 82, no. 82, pp. 177-186, 2018.

[19] A. Akpinar, G. B. Yaman, A. Demirdas, and S. Onal, "Possible role of adrenomedullin and nitric oxide in major depression," Progress in Neuro-Psychopharmacology and Biological Psychiatry, vol. 46, pp. 120-125, 2013.

[20] Q. A. Hussain, I. J. McKay, C. Gonzales-Marin, and R. P. Allaker, "Regulation of adrenomedullin and nitric oxide production by periodontal bacteria," Journal of periodontal research, vol. 50, no. 5, pp. 650-657, 2015.

[21] Q. A. Hussain, I. J. McKay, C. Gonzales-Marin, and R. P. Allaker, "Detection of adrenomedullin and nitric oxide in different forms of periodontal disease," Journal of Periodontal Research, vol. 51, no. 1, pp. 16-25, 2016.

[22] R. P. Allaker, B. E. Sheehan, D. C. McAnerney, and I. J. McKay, "Interaction of adrenomedullin and calcitonin gene-related peptide with the periodontal pathogenPorphyromonas gingivalis," FEMS Immunology and Medical Microbiology, vol. 49, no. 1, pp. 91-97, 2007.

[23] D. M. Glubb, P. C. McHugh, X. Deng, P. R. Joyce, and M. A. Kennedy, "Association of a functional polymorphism in the adrenomedullin gene $(A D M)$ with response to paroxetine," The pharmacogenomics journal, vol. 10, no. 2, pp. 126-133, 2010.

[24] D. Bergman, M. Halje, M. Nordin, and W. Engström, "Insulinlike growth factor 2 in development and disease: a minireview," Gerontology, vol. 59, no. 3, pp. 240-249, 2013.

[25] Y. W. Luo, Y. Xu, W. Y. Cao et al., "Insulin-like growth factor 2 mitigates depressive behavior in a rat model of chronic stress," Neuropharmacology, vol. 89, pp. 318-324, 2015.

[26] A. Córdova-Palomera, M. Fatjó-Vilas, C. Gastó, V. Navarro, M. O. Krebs, and L. Fañanás, "Genome-wide methylation study on depression: differential methylation and variable methylation in monozygotic twins," Translational Psychiatry, vol. 5, no. 4, p. e557, 2015.

[27] I. Pomytkin, J. P. Costa-Nunes, V. Kasatkin et al., "Insulin receptor in the brain: mechanisms of activation and the role in the CNS pathology and treatment," CNS Neuroscience \& Therapeutics, vol. 24, no. 9, pp. 763-774, 2018.

[28] H. S. Suh, M. L. Zhao, L. Derico, N. Choi, and S. C. Lee, "Insulin-like growth factor 1 and 2 (IGF1, IGF2) expression in human microglia: differential regulation by inflammatory mediators," Journal of Neuroinflammation, vol. 10, no. 1, 2013.

[29] J. Xie, Q. Li, X. H. Zhu, Y. Gao, and W. H. Zhao, "IGF2BP1 promotes LPS-induced $\mathrm{NF} \kappa \mathrm{B}$ activation and pro-inflammatory cytokines production in human macrophages and monocytes,"
Biochemical and Biophysical Research Communications, vol. 513, no. 4, pp. 820-826, 2019.

[30] E. R. Butelman, V. Yuferov, and M. J. Kreek, “ $\kappa$-opioid receptor/dynorphin system: genetic and pharmacotherapeutic implications for addiction," Trends in Neurosciences, vol. 35, no. 10 , pp. 587-596, 2012.

[31] R. Henriksson, C. M. Bäckman, B. K. Harvey et al., “_PDYN_, a gene implicated in brain/mental disorders, is targeted by REST in the adult human brain," Biochimica et Biophysica Acta, vol. 1839, no. 11, pp. 1226-1232, 2014.

[32] Y. L. Hurd, "Subjects with major depression or bipolar disorder show reduction of prodynorphin mRNA expression in discrete nuclei of the amygdaloid complex," Molecular Psychiatry, vol. 7, no. 1, pp. 75-81, 2002, PMID: 11803449.

[33] Y. Zhang, Y. Wang, Q. Xu, J. Zhu, and Y. Lin, "Molecular and expression characteristics of resistin (RETN) and its effects on the differentiation of intramuscular preadipocyte in goat," Animal biotechnology, vol. 32, no. 1, pp. 113-120, 2021.

[34] A. F. Carvalho, D. Q. Rocha, R. S. McIntyre et al., "Adipokines as emerging depression biomarkers: A systematic review and meta- analysis," Journal of psychiatric research, vol. 59, pp. 28-37, 2014.

[35] B. Weber-Hamann, J. Kratzsch, D. Kopf et al., "Resistin and adiponectin in major depression: the association with free cortisol and effects of antidepressant treatment," Journal of psychiatric research, vol. 41, no. 3-4, pp. 344-350, 2007.

[36] C. Hryhorczuk, S. Sharma, and S. E. Fulton, "Metabolic disturbances connecting obesity and depression," Frontiers in Neuroscience, vol. 7, no. 7, 2013.

[37] R. Subba, R. Sandhir, S. P. Singh, B. N. Mallick, and A. C. Mondal, "Pathophysiology linking depression and type 2 diabetes: psychotherapy, physical exercise, and fecal microbiome transplantation as damage control," European Journal of Neuroscience, vol. 53, no. 8, pp. 2870-2900, 2021.

[38] Z. Akram, Z. H. Rahim, T. B. Taiyeb-Ali et al., "Resistin as potential biomarker for chronic periodontitis: a systematic review and meta-analysis," Archives of oral biology, vol. 73, pp. 311-320, 2017.

[39] A. Devanoorkar, R. Kathariya, N. Guttiganur, D. Gopalakrishnan, and P. Bagchi, "Resistin: a potential biomarker for periodontitis influenced diabetes mellitus and diabetes induced periodontitis," Disease Markers, vol. 2014, Article ID 930206, 7 pages, 2014.

[40] E. J. Nestler, " $\Delta$ FosB: a transcriptional regulator of stress and antidepressant responses," European Journal of Pharmacology, vol. 753, no. 753, pp. 66-72, 2015.

[41] P. A. Gajewski, G. Turecki, and A. J. Robison, "Differential expression of FosB proteins and potential target genes in select brain regions of addiction and depression patients," PLoS One, vol. 11, no. 8, 2016.

[42] D. Zeng, S. He, C. Ma et al., "Network-based approach to identify molecular signatures in the brains of depressed suicides," Psychiatry research, vol. 294, p. 113513, 2020.

[43] L. Song, J. Yao, Z. He, and B. Xu, "Genes related to inflammation and bone loss process in periodontitis suggested by bioinformatics methods," BMC Oral Health, vol. 15, no. 1, p. 105, 2015. 